\section{Svetlana Gazhva ${ }^{1}$, Yulia Ibragimova ${ }^{1 凶}$ (1), Valentina Ryabova $\mathbb{B}^{\mathrm{D}}$, Yulia Gazhva ${ }^{1}$, Irina Belova ${ }^{1}$ (D), Anna Tochilina ${ }^{2}$ (D), Irina Solovyeva ${ }^{2}$ D, Vaan Aivazyan ${ }^{2}$, Natig Abdullaev ${ }^{2}$}

\author{
${ }^{1}$ Privolzhsky Research Medical University, Nizhny Novgorod; \\ ${ }^{2}$ Academician I.N. Blokhin Research Institute of Epidemiology and \\ Microbiology, Nizhny Novgorod, Russia

\section{yuliatalipova@mail.ru}

\begin{abstract}
The high virulence of periodontal pathogenic bacteria, progression of chronic periodontitis in patients with comorbidities not always treated efficiently with conventional therapies, may result in oral dysbiosis. In this connection, the development of new pathogenetically oriented treatment methods and their improvement is of both theoretical and practical interest to the dental community. AIM: to improve the algorithms for the treatment of periodontitis associated with comorbidities and to assess its effectiveness using an immobilized synbiotic developed by the authors. METHODS: clinical, microbiological, analytical, and statistical methods were used in our study. The study included clinical examination and treatment of 93 patients of both genders ( 37 female and 56 male patients) between 18 and 65 years old with moderate generalized chronic periodontitis. The results confirm the effectiveness of adding the proposed symbiotic to the treatment of chronic periodontitis with underlying gastrointestinal, endocrine and cardiovascular diseases in oral administration to restore the gut microbiota as well as applied locally in the periodontal pocket.
\end{abstract}

KEYWORDS - periodontitis, microbiocenosis, dysbiosis, probiotics.

\section{INTRODUCTION}

Periodontal diseases are the most common dental diseases in any age group: they occur in $40 \%$ of young dental patients, and $90 \%$ and $100 \%$ of middle-aged and elderly patients, respectively [1]. The inflammation of tissues is characterized by remaining undetected at early stages, developing chronically, having short remission periods and frequent relapses, progressing rapidly, and being difficult to treat $[2,3]$. In recent years, periodontal diseases have often developed in patients with comorbidities, thus exacerbating the underlying condition which in turn accelerates tooth loss, causing significant morphofunctional changes of the mastication apparatus $[4,12-14]$. Periodontal pathogens of red and orange complexes, characterized by an invasive growth process, form complex relationships with each other inside the microbiota and affect the immune system, causing systemic inflammatory and degenerative processes [5-7]. Because of that, the existing options for the treatment of periodontitis with underlying pathology are not always efficient as they do not take into account the pathogenesis of diseases. This is why the development of new pathogenetically-oriented methods for the treatment of periodontitis with underlying pathology and dysbiosis is a critical task, and the development of means for determining oral microbiota composition is a promising area of dentistry.

The objective of the study is to improve the algorithms of the combination treatment of periodontitis in patients with comorbidities.

\section{MATERIALS AND METHODS}

The study included the clinical examination and treatment of 93 patients of both genders ( 37 female and 56 male patients) between 18 and 65 years old with moderate generalized chronic periodontitis. The patients were divided into 2 groups according to the treatment regimen: the first group (control group) included 32 patients of both genders ( 15 female and 17 male patients) between 18 and 65 years old with moderate generalized chronic periodontitis and underlying chronic pathology (gastrointestinal, endocrine and cardiovascular diseases) who received only the basic treatment, including desensitizing, non-steroidal anti-inflammatory drugs and general tonics; the second group (treatment group) included 61 patients ( 25 female and 34 male patients) with moderate generalized chronic periodontitis, with similar age, gender and comorbidity distribution, who received not only the basic treatment but also the proprietary immobilized multistrain synbiotic "LB-complex L" 
(Patent No. 2441907, priority date: July 29, 2010, Certificate of State Registration No. RU.77.99.88.003 .E.002.522.06.18) - both for oral administration and local application.

"LB-complex L" is a fourth-generation probiotic supplement - a consortium of living antagonistic active strains of bifidobacteria ( 3 strains) and lactobacilli (3 strains) immobilized on zeolite (an enterosorbent). Zeolite is volcanic sediment and a unique mineral sorbent. In "LB-complex L", microorganisms and zeolite particles are in direct contact with each other, with bacteria forming biofilms on the crystal surface. The microorganisms are released in the intestine, with the sorbent acting as a detoxicating agent. The probiotics were administered orally, with a dosage of $10 \mathrm{ml} /$ day in one or two doses for 25 days, and locally - injected into a periodontal pocket with a dosage of $0,1-0,2 \mathrm{ml}$ and then covered with a Diplen-denta film, for 14 days.

The clinical examination and treatment of patients were based at Dentistry Department, Privolzhsky Research Medical University. The quantitative and qualitative analysis of gut and periodontal pocket microbiota was carried out at the Laboratory of Microbiome Analysis and Restoration, Academician Blokhin Research Institute of Epidemiology and Microbiology (Nizhny Novgorod, Russia).

\section{Research methods:}

clinical, microbiological, analytical, and statistical methods.

The examination of a periodontal patient included standard examination with a periodontal probe, assessment of periodontal indices, and filling the patient's medical record. Experts assessed the periodontitis condition before the treatment, immediately after the treatment course, and 6 months later.

Oral hygiene was assessed using the simplified oral hygiene index (Green-Vermillion, 1964). Bleeding was measured using the Muhlemann-Son Sulcus Bleeding Index (SBI) which is based on assessing bleeding from the gingival sulcus on probing. The degree of inflammation was visualized by calculating the associated periodontal index (API). The API is designed by the study authors and is based on assessing the following factors: gum inflammation, sulcus bleeding on probing, the depth of periodontal pockets, the degree of tooth mobility, and gum recession.

The quantitative and qualitative assessment of the gut biocenosis was conducted using the unified methodology of colon microbiota assessment and evaluation $[10,11]$.

The quantitative and qualitative assessment of the periodontal pocket microbiocenosis was conducted according to the following procedure: No oral hygiene procedures were conducted before the sampling. The material was sampled from the periodontal pocket using a single-use polystyrene sterile inoculating loop No. 2 in the volume of $1 \mu \mathrm{l}(0.001 \mathrm{ml})$ and a needle produced by Nuova Aptaca SRL, Italy. After that, the material was suspended in Eppendorf tubes with $0.09 \mathrm{ml}$ of Haenel buffer solution for obtaining a $10^{-2}$ dilution, and the loop was placed into a sterile polymer test tube with an anaerobic transport medium (Stuart medium, China). The material was delivered to a laboratory within 2 hours of being taken. The $10^{-2}$ solution was used for inoculation on plating media: chocolate agar, Endo-GRM agar, Enterococcus Agar, Sabouraud agar No. 2 produced by State Research Center for Applied Microbiology and Biotechnology, Obolensk, Russia.

The inoculation was performed according to the following procedure: the material was spread with a $3 \mathrm{~mm}$ loop in the A sector, then the loop was flamed, and four linear streaks were made from sector $A$ to sector I. The same method with loop flaming was used for re-inoculation from sector I to sector II, and from sector II to sector III [8].

The inoculants were incubated aerobically at $37 \pm 1^{\circ} \mathrm{C}$ for $24-48$ hours.

The same method was used for the inoculation of the $10^{-2}$ solution on Schaedler agar (BBLTM Schaedler agar, Becton Dickison, USA), Clostridial agar (M497 HiMedia Laboratories Pvt. Limited, India) and agar MRS-4 (Pharmacotherapy Research and Development Center, Saint Petersburg). Specimens were inoculated from the anaerobic transport medium to a bile esculin plating medium with the introduction of a selective supplement for anaerobic bacteria - Bacteroides Bile Esculin Agar (M805; FD062 HiMedia Laboratories Pvt. Limited, India), Schaedler agar, Clostridial agar, and MRS-4. The inoculants were incubated anaerobically at $37 \pm 1^{\circ} \mathrm{C}$ for $72-96$ hours. Incubation was performed using the GasPak Anaerobe Gas Generation Pouch System with Indicator (Becton Dickinson, USA).

After the incubation, the number of bacteria colonies was counted in each sector, with the identification of 4-5 colonies of each species. For bacteria identification, a Bruker Autoflex speed MALDI-TOF mass spectrometer was used with Biotyper software. The preparation of daily culture samples was conducted according to the standard direct deposition method presented in the user manual: a sample was applied to three target cells, a matrix solution was deposited ( $\alpha$-cyano- 4 -hydroxycinnamic acid $(\alpha$-CHCA)) and dried up, and then combined mass spectra were obtained automatically for each cell. The mass spectra 
were identified, recorded, processed, and analyzed using the BioTyper RTC software. The reliability of identification was determined by the score values $(2,000-$ 3,000 - species identification, 1,999-1,700 - genus identification, 1,699-0 - not reliable identification) and consistency categories (A - species consistency, $\mathrm{B}-$ genus consistency, $\mathrm{C}-$ no consistency.

The number of identified microorganisms of each species was counted according to Table 1 .

\section{Table 1. Assessment of the microbial content of periodontal pockets}

\begin{tabular}{|c|c|c|c|c|}
\hline \multicolumn{4}{|c|}{$\begin{array}{l}\text { Number of colonies of each isolated microor- } \\
\text { ganism species in sectors }\end{array}$} & \multirow{2}{*}{$\begin{array}{l}\text { Amount of each type } \\
\text { of microorganism in } 1 \\
\mathrm{ml} \text { of separable gingi- } \\
\text { val pocket, CFU/ml }\end{array}$} \\
\hline A & I & ॥ & III & \\
\hline $1-6$ & - & - & - & $<10^{4}$ \\
\hline $8-20$ & - & - & - & $3 \cdot 10^{4}$ \\
\hline $20-30$ & - & - & - & $5 \cdot 10^{4}$ \\
\hline $30-60$ & - & - & - & $1 \cdot 10^{5}$ \\
\hline $70-80$ & - & - & - & $5 \cdot 10^{5}$ \\
\hline $100-150$ & $5-10$ & - & - & $1 \cdot 10^{6}$ \\
\hline Can't count & $20-30$ & - & - & $5 \cdot 10^{6}$ \\
\hline Can't count & $40-60$ & - & - & $1 \cdot 10^{7}$ \\
\hline
\end{tabular}

The quantitative and qualitative characteristics and the dysbiosis of periodontal pocket microbiocenosis were assessed according to an article by N.A. Sakharuk [9].

The treatment included the removal of supragingival and subgingival calculus, oral hygiene instruction and control, gingival curettage (if medically required), the correction of traumatic occlusion, the splinting of mobile teeth, and antiseptic treatment (using chlorhexidine $0.05 \%$ antiseptic solution).

\section{RESULTS}

The clinical examination and index assessment showed poor oral hygiene, high Muhlemann-Son Sulcus Bleeding Index, and API index in $98 \%$ of patients with moderate generalized chronic periodontitis.

The pre-treatment analysis of oral hygiene assessment in patients with moderate generalized chronic periodontitis showed mineralized and nonmineralized dental plaque. The oral hygiene index was $3.8 \pm 0.08$ points for the control group patients, and $4.1 \pm 0.06$ points for the treatment group patients, which indicates poor oral hygiene in all patients. Immediately after the periodontitis treatment, oral hygiene instruction and control, the oral hygiene of patients in both groups improved significantly - to $0.6 \pm 0.07$ points in group 1 and $0.8 \pm 0.07$ in group 2 , which indicates good oral hygiene. The check-up examination 6 months after the treatment showed a slight worsening of the oral hygiene level. Still, the average oral hygiene level of the treatment group patients is considered good even though it is close to the satisfactory level ( $1.2 \pm 0.04$ points), and the level of the control group patients is considered satisfactory (1.6 \pm 0.03 points).

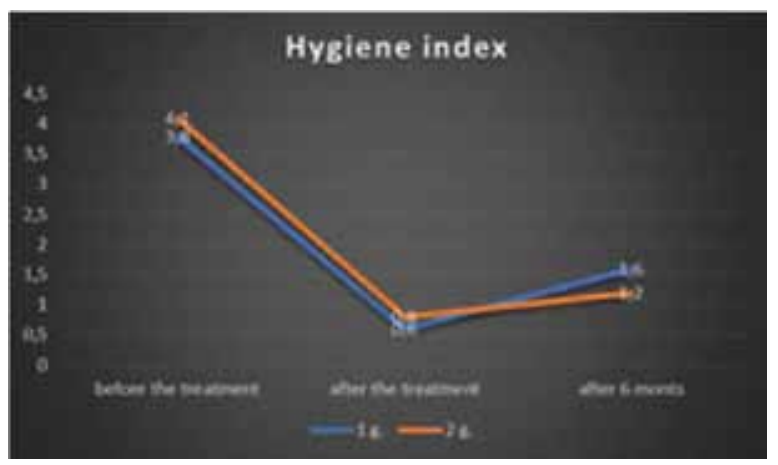

Diagram 1. Comparative assessment of the hygiene index

The condition of periodontal tissue improved, including a reduction in bleeding determined by the Muhlemann-Son Sulcus Bleeding Index. Before the treatment, the treatment group patients showed bleeding of high severity $-2.1 \pm 0.04$ points and the control group patients had bleeding of moderate severity $1.9 \pm 0.03$ points. After the periodontal treatment, the level of bleeding decreased in both groups: to $1.1 \pm 0.04$ points in group 1 and $1.3 \pm 0.02$ points in group 2 , which indicates bleeding of moderate severity. The check-up examination after 6 months indicated good tissue condition among the treatment group patients $-0.8 \pm 0.05$ points, and bleeding of moderate severity among the control group patients $-1.3 \pm 0.04$ points.

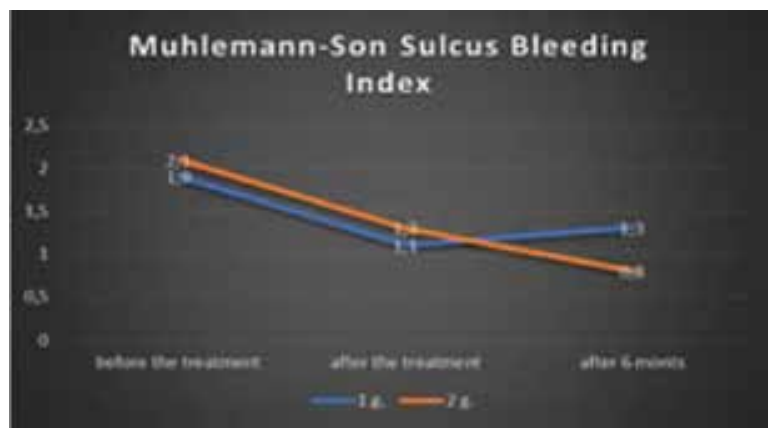

Diagram 2. Comparative assessment of the Muhlemann-Son Sulcus Bleeding Index 
The pre-treatment associated periodontal index was $8.7 \pm 0.09$ in the first group and $9.2 \pm 0.12$ points in the second group, which indicates moderate severity of periodontal disease, although getting closer to high severity in the second group. After the treatment, the API decreased to $7.6 \pm 0.08$ points in the first group and $7.9 \pm 0.10$ in the second group. Six months later, the API in both groups decreased - to $7.1 \pm 0.11$ points in the control group and to $6.6 \pm 0.09$ in the treatment group which indicates moderate periodontitis. However, the fact that the average API in the treatment group is closer to the lower boundary of moderate severity, can be considered as a sign that there is no inflammation or at least that it has decreased significantly.

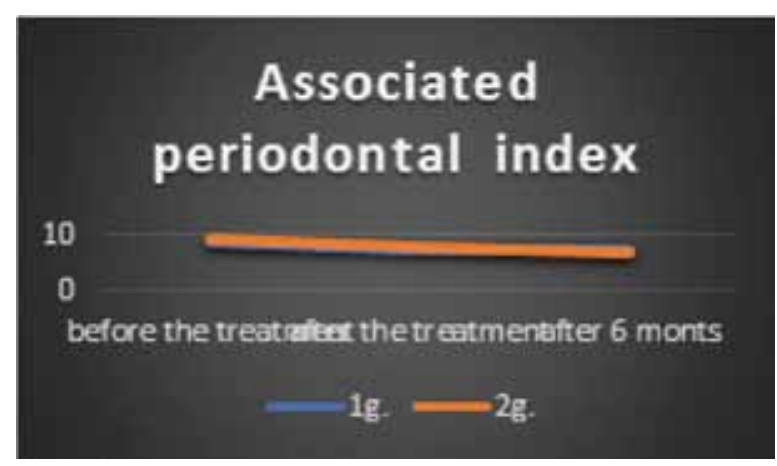

Diagram 3. Comparative assessment of the associated periodontal index

Before the treatment, bifidobacteria were present in the fecal microbiota of $100 \%$ of patients from both groups, with $37.5 \%$ of patients having a low level of bifidobacteria: $10^{6}-10^{7} \mathrm{CFU} / \mathrm{ml}$. The most prevalent species were B.longum (75\%), B.adolescentis (62.5\%), and B.bifidum (33.3\%). B.dentium, B.pseudocatenulatum, B.animalis, B.catenulatum, B.angulatum were detected in individual cases. Bacteria of the Lactobacillaceae family were detected only in $70.8 \%$ of patients, with a concentration of less than $10^{7} \mathrm{CFU} / \mathrm{ml}$ in $29.1 \%$ of patients. $21 \%$ of patients had no lactobacilli in their colon microbiota. Most often, the following species were detected in fecal microbiota: L.gasseri (45.8\%), L.paracasei и L. vaginalis (29.1\%) L.oris (25\%), L.crispatus and L.salivarius (20.8\%). Bacteria of other genera and species were detected in individual cases. The quantity Bacteroides spp. was low in $91.6 \%$ of patients, at the level of $10^{8} \mathrm{CFU} / \mathrm{ml}$ - in $41,6 \%$. B. uniformis and B.ovatus (33.3\%), B.vulgatus and B.thetaiotaomicron (25\%) were the most prevalent species. E.coli was detected in all patients, with a low concentration of $106 \mathrm{CFU} / \mathrm{ml}$ and less in $41.6 \%$ of patients. Lactose-negative coliform bacteria were detected in $4.2 \%$ of patients. Enterococci were detected in $50 \%$ of patients $\left(10^{5}-10^{8} \mathrm{CFU} / \mathrm{ml}\right)$. Most often, E.faecalis (33.3\%) and E.faecium (16.6\%) were detected in fecal microbiota. Various species of the Clostridium genus were identified in $52.6 \%$ of patients at concentrations within $10 \mathrm{CFU} / \mathrm{ml}$ which is considered normal. In individual cases, higher concentrations of C.innocuum $\left(10^{6} \mathrm{CFU} / \mathrm{ml}\right)$ and C.perfringens $\left(10^{7} \mathrm{CFU} / \mathrm{ml}\right)$ were identified. S.aureus was identified in $12.5 \%$ of patients. Coagulase-negative staphylococci were found in $41.2 \%$ of patients, with a concentration of over $10^{5} \mathrm{CFU} / \mathrm{ml}$ in $12.5 \%$ of patients. Significant levels of opportunistic pathogenic Enterobacterales $\left(>10^{5} \mathrm{CFU} / \mathrm{ml}\right)$ were identified in $45.8 \%$ of patients, most often - Enterobacter cloaceae (16.6\%) and Klebsiella pneumoniae (12.5\%). Proteus mirabilis, M.morganii, Raoultella ornithinolytica, and Citrobacter freundii were detected less often and in lower quantities. Yeast-like fungi of the Candida genus were identified in the colon microbiota of $70.8 \%$ of patients. The most prevalent species (with concentrations of $10^{5}-10^{7} \mathrm{CFU} / \mathrm{ml}$ ) were C.kefyr and C.lusitaniae. C.albicans was identified more often - in 54.2\% of patients but at lower levels of $10^{2}-10^{4} \mathrm{CFU} / \mathrm{ml}$. C.crusei, C.tropicalis, C.guillermondii, C.parapsilosis, C.dublinensis, and C.glabrata were identified in individual cases. Non-fermenting gram-negative bacilli were detected in significant quantities $\left(>10^{5}\right.$ $\mathrm{CFU} / \mathrm{ml}$ ) in individual cases, including P.aeruginosa, Acinetobacter lwoffii, and Comamonas testosteroni. The following Streptococcus species were detected in significant quantities $\left(10^{6}-10^{7} \mathrm{CFU} / \mathrm{ml}\right)$ : S.lutetiensis, S.pleomorphus, S.salivarius, S.sanguinis, S.gallolyticus, S.vestibularis, and S.parasanguinis, as well as Collinsella aerofaciens, Eggerthella lenta, and Streptomyces lavendulae. It should be noted that although the above-mentioned Streptococcus species were only identified in individual cases, some species of Streptococcus bacteria were detected in $45.8 \%$ of patients. Pre-treatment assessment of the overall colon microbiota condition showed grade I dysbiotic disorders in $50 \%$ of patients, grade II disorders in $33.3 \%$ of patients, grade III disorders in $8.3 \%$ of patients, and normal microbiocenosis in $8.3 \%$ of patients.

After the treatment, control group patients did not show any positive dynamics in restoring the microbiocenosis. Having undergone the combination treatment, the treatment group patients showed an increase in bifidobacteria and lactobacilli in colon microbiota to $10^{8}-10^{9} \mathrm{CFU} / \mathrm{ml}$ in $94.7 \%$ and $100 \%$ cases, respectively. Bacteroides spp. was detected in 100\% of patients, at the level of $108 \mathrm{CFU} / \mathrm{ml}$ - in $73.6 \%$ of patients. Opportunistic microorganisms of the Enterobacterales order and Staphylococcaceae family were detected in significant quantities $\left(>10^{5} \mathrm{CFU} / \mathrm{ml}\right)$ 
in $15.7 \%$ of the treatment group patients and $45.8 \%$ of the control group patients. After the treatment, yeastlike fungi of the Candida genus were only identified in $47.3 \%$ of the treatment group patients, while no such positive dynamics were observed in the control group $(70.8 \%)$. After the treatment, microbiocenosis recovered in $87.5 \%$ of the treatment group patients, with improvements in the condition of the remaining 12.5\%: pronounced grade III and II dysbiosis disorders improved to mild grade I disorders.

Pre-treatment bacteriological examination of the periodontal pocket showed that S.aureus was detected in the quantities of $10^{3}-10^{6} \mathrm{CFU} / \mathrm{ml}$ in $16.6 \%$ of patients of both groups. Coagulase-negative staphylococci were found in $66.6 \%$ of patients. The most common bacterium is S.epidermidis (37,5\%), the ones present in the largest quantity (105-106 $\mathrm{CFU} / \mathrm{ml}$ ) were S.epidermidis, S.warneri, S.xylosus, and S.felis. Neisseria were detected in $87.5 \%$ of patients in the amount of $10^{3}-10^{6} \mathrm{CFU} / \mathrm{ml}$. The most prevalent of the 12 identified Neisseria species were N.mucosa (33.3\%), N.flavescens and N.elongata (29.1\%), as well as N.macacae (25\%). The genus represented by the largest number of species was Streptococcus, with 21 species identified. In most cases, S.oralis (83.3\%), S.sanguinis (66.6\%), S.vestibularis (62.5\%), S.pneumoniae (58.3\%), S.anginosus (54.1\%), and S.salivarius (50\%) were identified. S.sanguinis, S.cristatus, S.psudopneumoniae, and S.gordonii were detected in the largest quantities $\left(10^{7} \mathrm{CFU} / \mathrm{ml}\right)$. Bacteria of the Lactobacillaceae family (17 species) were mostly detected at the level of $10^{3} \mathrm{CFU} / \mathrm{ml}$. In most cases, L.gasseri (25\%), L.paracasei (16.6\%), and L.oris (12.5\%) were identified. Haemophilus bacteria were detected in $16.6 \%$ of patients in the amount of $10^{4}-10^{7} \mathrm{CFU} / \mathrm{ml}$. Yeast-like fungi of the Candida genus were identified in $25 \%$ of patients. C.albicans, C.lambica, C. lusitaniae, and C.kefyr were detected equally often in the quantities of 105-107 CFU/ $\mathrm{ml}$. Bacteria of the Veillonella genus were detected in $95.8 \%$ of patients. V.parvula was identified in $66.6 \%$ of patients, V.atypica - in $29,2 \%$ of patients. Two-thirds of the Veillonella spp. identified in the periodontal pocket were present in the quantities of $10-10^{4} \mathrm{CFU} / \mathrm{ml} .12$ species of the Prevotella genus were detected, with P.nigrescens, P.oralis, and P.denticola present in the quantities of $10^{5} \mathrm{CFU} /$ $\mathrm{ml}$. The quantity of other Prevotella species was in the range between 10 and $10^{3} \mathrm{CFU} / \mathrm{ml}$. Among the Actinomyces genus bacteria, the most common species were A.naeslundii (37.5\%), A.odontolyticus (29.2\%), and A.oris (25\%), with a wide range of quantity between 10 and $107 \mathrm{CFU} / \mathrm{ml}$. 45.8\% of specimens contained Fusobacterium nucleatum in the quantity of $10^{3}-10^{5} \mathrm{CFU} / \mathrm{ml}$. Also, Selenomonas spp. was identified in $25 \%$ of patients, mostly in the form of S.noxia and in the amount of $105 \mathrm{CFU} / \mathrm{ml}$; single Clostridium spp. cells - in $20.8 \%$ of patients; Rothia mucilaginosa in the quantities of $10^{3}-10^{5} \mathrm{CFU} / \mathrm{ml}$ — in 16.6\% of patients, and Capnocytophaga spp. in the quantities between 10 and $105 \mathrm{CFU} / \mathrm{ml}$ - in $16.6 \%$ of patients. Other bacteria (over 40 genera, each including between one and four species) were detected in individual patients, forming their individual microbiota profiles.

Pre-treatment assessment of the overall periodontal pocket microbiota condition showed grade I dysbiotic disorders in $29.2 \%$ of patients, grade II disorders in $45.8 \%$ of patients, and grade III disorders in $25 \%$ of patients.

After the basic treatment, $15.7 \%$ of the control group patients showed normal microbiota condition, $59.2 \%$ - grade I dysbiotic disorders, and 25\% grade II dysbiotic disorders, with no patients having grade III dysbiotic disorders.

After the treatment, the number of treatment group patients with staphylococci decreased. S.aureus was identified in $10.5 \%$ of patients, with its concentration not exceeding $10^{4} \mathrm{CFU} / \mathrm{ml}$. The number of coagulase-negative staphylococci decreased, with only S.capitis and S.epidermidis detected in the quantities of $10^{3}-10^{4} \mathrm{CFU} / \mathrm{ml}$. The most common Neisseria species were N.flavescens (42.1\%), N.mucosa (26.3\%), and N.elongata (26.3\%). The occurrence of Streptococci slightly decreased: S.oralis was identified in $73.6 \%$ of patients, S.pneumoniae - in $68.4 \%$ of patients, S.salivarius and S.vestibularis - in $63.1 \%$ of patients, S.anginosus - in $47.3 \%$ of patients, and S.sanguinis - in $42.1 \%$ of patients. Streptococcus species were identified in various quantities in the range between 10 and $10^{6} \mathrm{CFU} / \mathrm{ml}$. The number of Lactobacilli species identified in the periodontal pocket in the quantities of $10^{5}-10^{6} \mathrm{CFU} / \mathrm{ml}$ increased. L.gasseri, L.paracasei, L.rhamnosus, L.plantarum, L.oris, L.amylovorus, L.frumentii, and L.fermentum were detected with such titers. Haemophylus parainfluenzae was identified rarer (5.2\%) and in lower quantities $\left(10^{3} \mathrm{CFU} / \mathrm{ml}\right)$. After the treatment, the substrate of only $5.2 \%$ of patients showed single C.albicans cells. Other species of the Candida genus were not detected. The occurrence, quantities, and species composition of the identified Veillonella and Prevotella remained almost the same. After the treatment, the most common Actinomyces species was A.oris (36.8), with A.naeslundii detected only in $10.5 \%$ of patients. The quantity of identified Actinomyces did not exceed $10^{6} \mathrm{CFU} / \mathrm{ml}$. After the treatment, Selenomonas spp. was identified in $42.1 \%$ of patients in the quantity of $10^{5}-10^{7} \mathrm{CFU} / \mathrm{ml}$. The 
occurrences and quantities of Capnocytophaga spp. also decreased after the treatment. Post-treatment assessment of the overall periodontal pocket microbiota condition showed grade I dysbiotic disorders only in $26.3 \%$ of the treatment group, with normal microbiological indicators in all the other patients in that group (73.7\%).

After the treatment, the periodontal pocket microbiocenosis became normal in $73.7 \%$ of the treatment group patients, with the microbiota condition also improved in the remaining $26.3 \%$ of patients from grade I and II dysbiosis to compensated dysbiosis.

Before the treatment, combined intestinal and periodontal pocket dysbiosis was identified in $83.3 \%$ of patients, with $16.6 \%$ showing periodontal pocket dysbiosis and normal intestinal microbiota. After the treatment, only $15.7 \%$ of the control group patients showed normalization of the intestinal and periodontal pocket microbiocenosis, compared to $73.7 \%$ of the treatment group patients. The treatment groups also showed positive dynamics of microbiocenosis recovery in the remaining $26.3 \%$ of cases.
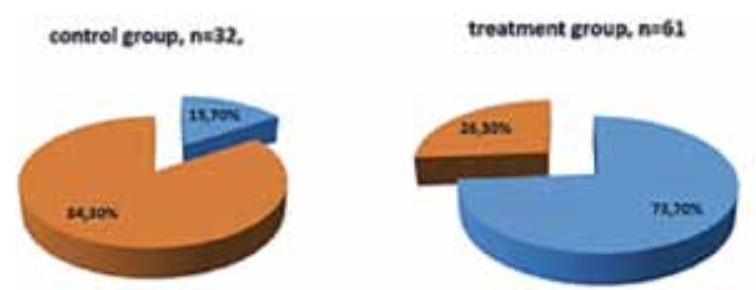

$=N * N-$ normalization of the intestinal and periodontal pocket microbiocenosis.

$=0 * 0$ - the dnbiosis of the intestinal and perlodontal pocket microbiota

Fig. 1. The post-treatment condition of the intestinal and periodontal pocket microbiota in patients with generalized chronic periodontitis and comorbidities (in two groups)

\section{CONCLUSION}

When added to the diet as part of the combination basic treatment of patients with moderate generalized chronic periodontitis and comorbidities, the proprietary immobilized synbiotic "LB-complex L" allowed to achieve sustained periodontal disease remission, to counterbalance the effect of inflammation on the development of periodontitis, and to restore the gut microbiocenosis.

\section{REFERENCES}

1. Gazhva S. I. Surgical periodontal treatment: Recommended by the Educational and methodological association of Russian universities on medical and pharmaceutical education as a textbook for postgraduate dentistry courses / Nizhny Novgorod: Nizhny Novgorod State Medical Academy, 2003. - 110 p. ISBN 5703204933.

2. GazhVA S. I. A comprehensive study of the mucous membrane of the tongue dorsum aimed at diagnosing a range of medical conditions and personal identification: area of specialization No. 14.01.14 "Dentistry": abstract of a Doctore of Medicine thesis / Moscow, 2000. -26 p.

3. GazhVa S. I., Voronina A. I., Shkarednaya O. V. / Analyzing the clinical and immunological status of the oral cavity in patients with mild and moderate generalized chronic periodontitis when undergoing antibacterial therapy / Dentistry. - 2010. - Vol. 89. No. 3. - P. 30-33.

4. Gazhya S. I., Pillipenko K. I., Shkarednaya O. V., Menshikova Yu. V./ The clinical efficacy of various medications in the conservative therapy of generalized chronic periodontitis / / Clinical dentistry. - 2011. - No. 3(59). - P. 34-36.

5. Gazhya S. I., Shkarednaya O. V., Pyatova Ye. D. / A comprehensive approach to the treatment of oral mucosal diseases in patients with chronic gastritis / Dentistry. - 2013. - Vol. 92. - No. 6. - P. 16-19.

6. Gazhva S. I., Shmatova S. O., Goryacheva T. P., KHudoshin S. V. / Improving the methods of mild and moderate periodontitis treatment with the use of PDT and laser pocket disinfection / // Modern problems of science and education. - 2014. - No. 6. P. 1070.

7. Muraev A. A., Gazhva Y. V., Ryabova V. M. [eT AL.] / Development and preclinical studies of Orthotopic Bone Implants Based on a Hybrid Construction from poly(3-hydroxybutyrate) and sodium alginate / // Modern Technologies in Medicine. - 2016. - Vol. 8. - No 4. - P. 42-50. - DOI 10.17691/stm2016.8.4.06.

8. Pravosudova N. A., Melnikov V. L. Oral microbiology / A textbook for medicine students. - 2013. $-89 \mathrm{p}$.

9. SAKHARUK N. A. Normal and pathological oral microbiota. The morphology of the Candida genus fungi // Bulletin of the Voronezh State Medical University. - 2008. - Vol. 7, No. 2. - P. 137-143.

10. Tochilina A. G., Criteria for the assessment of the colon microbiocenosis composition / I. V. Belova, I. V. Solovyeva [et al.] // The guide of a clinical diagnostic laboratory manager. - 2016. - No. 8. - P. 54-78.

11. Industry Standard OST 91500.11.0004- 2003 "Patient management guidelines. Intestinal dysbacteriosis" (approved by Order of the Ministry of Health of the Russian Federation No. 231 of June 9, 2003).

12. Ostrovskaya L.Yu., Beybulatova D., ZakhaRova N.B. Gingival fluid as a potential object for diagnostics process. Archiv EuroMedica. 2020. Vol. 10; 2: 104-106. https://dx.doi.org/10.35630/2199$885 \mathrm{X} / 2020 / 10 / 2.27$ 
13. Karpyuk V.B., Perova M.D., Gilevich I.V. Cellpotentiated regenerative technologies for restoring jaw bone tissues in case of odontogenic inflammatory \& destructive process // Archiv EuroMedica. 2019. Vol. 9. № 2. P. 140-146. https://doi.org/10.35630/2199$885 \mathrm{X} / 2019 / 9 / 2 / 140$

14. Ostrovskaya L.Yu., Eremin O.V., Zakharova N.B. Gum fluid biomarkers in personalized diagnostics of inflammatory periodontal diseases. Archiv EuroMedica. 2021. Vol. 11; 4: 130-135. https://dx.doi. org/10.35630/2199-885X/2021/11/4.30

15. Riep B, Edesi-Neuss L, Claessen F, Skarabis H, EHMKe B, FLEMmig TF, BERNIMOULIN JP, GÖBEL UB, Moter A. Are putative periodontal pathogens reliable diagnostic markers? J Clin Microbiol. 2009 Jun;47(6):1705-11. Epub 2009 Apr 22.

16. Henderson B., Wilson M., Sharp L. AND Ward J.M. HeNderson B., Wilson M., SharP L. Actinobacillus actinomycetemcomitans REVIEW ARTICLE.J. Med. Microbiol. - Vol. 51 (2002), 1013-1020. https://doi.org/10.1099/0022-1317-51$12-1013$
17. In vitro susceptibility of Candida albicans isolates from apical and marginal periodontitis to common antifungal agents/T. M. Waltimo, D. Orstavik, J. H Meurman, L. P. Samaragayake //J. Oral. Microbiol. Immunol. - 2000. - Vol. 15, No 4. - P. 245-248.

18. Al-Zahrani M.S., Kayal R.A., BissadaN.F. Periodontitis and cardiovascular disease: a review of shared risk factors and new findings supporting a causality hypothesis. Quintessence Int 2006;37:1:11-18.

19. Diomedi M., Leone G., Renna A. The role of chronic infection and inflammation in the pathogenesis of cardiovascular and cerebrovascular disease. TimelyTopMedCardiovascDis 2006;1:10:6. 